\title{
New pseudorandom sequences constructed using multiplicative inverses
}

by

Huaning Liu (Xi'an)

1. Introduction. In a series of papers Mauduit, Rivat and Sárközy (partly with other authors) studied finite pseudorandom binary sequences

$$
E_{N}=\left\{e_{1}, \ldots, e_{N}\right\} \in\{-1,+1\}^{N} .
$$

In [5] Mauduit and Sárközy first introduced the following measures of pseudorandomness: the well-distribution measure of $E_{N}$ is defined by

$$
W\left(E_{N}\right)=\max _{a, b, t}\left|\sum_{j=0}^{t-1} e_{a+j b}\right|,
$$

where the maximum is taken over all $a, b, t \in \mathbb{N}$ with $1 \leq a \leq a+(t-1) b \leq N$. The correlation measure of order $k$ of $E_{N}$ is

$$
C_{k}\left(E_{N}\right)=\max _{M, D}\left|\sum_{n=1}^{M} e_{n+d_{1}} e_{n+d_{2}} \cdots e_{n+d_{k}}\right|,
$$

where the maximum is taken over all $D=\left(d_{1}, \ldots, d_{k}\right)$ and $M$ with $0 \leq$ $d_{1}<\cdots<d_{k} \leq N-M$, and the combined (well-distribution-correlation) $P R$-measure of order $k$

$$
Q_{k}\left(E_{N}\right)=\max _{a, b, t, D}\left|\sum_{j=0}^{t} e_{a+j b+d_{1}} e_{a+j b+d_{2}} \cdots e_{a+j b+d_{k}}\right|
$$

is defined for all $a, b, t, D=\left(d_{1}, \ldots, d_{k}\right)$ with $1 \leq a+j b+d_{i} \leq N(i=$ $1, \ldots, k)$. In [6] the connection between the measures $W$ and $C_{2}$ was studied.

A pseudorandom sequence $E_{N}$ is considered to be "good" if both $W\left(E_{N}\right)$ and $C_{k}\left(E_{N}\right)$ (at least for small $k$ ) are "small" in terms of $N$. Later Cas-

2000 Mathematics Subject Classification: Primary 11K45.

Key words and phrases: pseudorandom, binary sequence, exponential sums.

Supported by the National Natural Science Foundation of China under Grant No. 60472068; and the Natural Science Foundation of the Education Department of Shaanxi Province of China under Grant No. 06JK168. 
saigne, Mauduit and Sárközy [3] proved that this terminology is justified since for almost all $E_{N} \in\{-1,+1\}^{N}$, both $W\left(E_{N}\right)$ and $C_{k}\left(E_{N}\right)$ are less than $N^{1 / 2}(\log N)^{c}$. Moreover, it was shown in [5] that the Legendre symbol forms a "good" pseudorandom sequence. In [1] and [2], Cassaigne and coauthors studied the pseudorandomness of the Liouville function, defined as $\lambda(n)=(-1)^{\Omega(n)}(\Omega(n)$ being the number of prime factors of $n$ counted with multiplicity) and also of $\gamma(n)=(-1)^{\omega(n)}(\omega(n)$ being the number of distinct prime factors of $n)$. Furthermore, let

$$
K(m, n ; p)=\sum_{a=1}^{p-1} \mathrm{e}\left(\frac{m a+n \bar{a}}{p}\right)
$$

denote the Kloosterman sums, where $\mathrm{e}(y)=e^{2 \pi i y}, p$ is a prime, and $\bar{a}$ is the multiplicative inverse of $a$ modulo $p$ such that $1 \leq \bar{a} \leq p-1$. Fouvry (with coauthors) [4] showed that the signs of $K(1, n ; p)$ form a "good" pseudorandom binary sequence.

Assume that $p$ is a prime number, $f(x) \in \mathbb{F}_{p}[x]$ has degree $k(0<k<p)$ and no multiple zero in $\overline{\mathbb{F}}_{p}$, and $r_{p}(n)$ is defined to be the least non-negative residue of $n$ modulo $p$. Define the binary sequence $E_{p}=\left(e_{1}, \ldots, e_{p}\right)$ by

$$
e_{n}= \begin{cases}+1 & \text { if }(f(n), p)=1 \text { and } r_{p}(\overline{f(n)})<p / 2, \\ -1 & \text { if either }(f(n), p)=1 \text { and } r_{p}(\overline{f(n)})>p / 2, \text { or } p \mid f(n) .\end{cases}
$$

Mauduit and Sárközy [7] proved that this large family of finite binary sequences has strong pseudorandom properties.

As was said in [5], the search for new approaches and new constructions should be continued. The purpose of this paper is to give some new examples of pseudorandom sequences. Define

$$
e_{n}= \begin{cases}(-1)^{\bar{n}+\overline{n+x}} & \text { if } p \nmid n \text { and } p \nmid n+x, \\ 1 & \text { otherwise, }\end{cases}
$$

where $x$ is an integer with $1 \leq x \leq p-1$. We shall prove that $\left\{e_{n}\right\}$ is a "good" pseudorandom sequence:

Theorem 1.1. Let $p$ be an odd prime, and let $E_{p-1}=\left\{e_{1}, \ldots, e_{p-1}\right\}$ be defined by (1.1). Then

$W\left(E_{p-1}\right) \ll p^{1 / 2} \log ^{3} p, \quad C_{2}\left(E_{p-1}\right) \ll p^{1 / 2} \log ^{5} p, \quad Q_{2}\left(E_{p-1}\right) \ll p^{1 / 2} \log ^{5} p$.

2. Some lemmas. We need the following lemmas.

Lemma $2.1([9])$. Let $p$ be a prime, and $m$ and $n$ be integers. Then

$$
K(m, n ; p) \ll(m, n, p)^{1 / 2} p^{1 / 2},
$$

where $(m, n, p)$ denotes the greatest common divisor of $m, n$ and $p$. 
Lemma $2.2([8])$. For any polynomials $g(x), h(x) \in \mathbb{F}_{p}[x]$ such that the rational function $f(x)=g(x) / h(x)$ is not constant on $\mathbb{F}_{p}$, let $s$ be the number of distinct roots of $h(x)$. Then

$$
\left|\sum_{\substack{n \in \mathbb{F}_{p} \\ h(n) \neq 0}} \mathrm{e}\left(\frac{g(n)}{h(n) p}\right)\right| \leq(\max (\operatorname{deg}(g), \operatorname{deg}(h))+s-1) \sqrt{p} .
$$

Lemma 2.3. For $1 \leq a, b, x, r, s \leq p-1$ and $1 \leq u \leq p$, we have

$$
\Psi=\sum_{\substack{j=0 \\ p \nmid a+j b \\ p \nmid a+j b+x}}^{p-1} \mathrm{e}\left(\frac{r \overline{a+j b}+s \overline{a+j b+x}+u j}{p}\right) \ll \sqrt{p} .
$$

Proof. If $u=p$, from the properties of a residue system we get

$$
\begin{aligned}
\Psi & =\sum_{\substack{j=0 \\
p \nmid a+j b \\
p \nmid a+j b+x}}^{p-1} \mathrm{e}\left(\frac{r \overline{a+j b}+s \overline{a+j b+x}}{p}\right)=\sum_{\substack{j=0 \\
p \nmid a+j \\
p \nmid a+j+x}}^{p-1} \mathrm{e}\left(\frac{r \overline{a+j}+s \overline{a+j+x}}{p}\right) \\
& =\sum_{\substack{j=1 \\
p \nmid j+x}}^{p-1} \mathrm{e}\left(\frac{r \bar{j}+s \overline{j+x}}{p}\right) .
\end{aligned}
$$

Since

$$
\overline{j+x} \equiv \overline{j x \bar{x}+x j \bar{j}} \equiv \bar{x} \bar{j} \overline{\bar{x}+\bar{j}} \equiv \bar{x}(\bar{j}+\bar{x}-\bar{x}) \overline{\bar{j}+\bar{x}} \equiv \bar{x}-\bar{x}^{2} \overline{\bar{j}+\bar{x}} \bmod p,
$$

by Lemma 2.1 we have

$$
\begin{aligned}
\Psi & =\sum_{\substack{j=1 \\
p \nmid \bar{j}+\bar{x}}}^{p-1} \mathrm{e}\left(\frac{r \bar{j}+s\left(\bar{x}-\bar{x}^{2} \overline{\bar{j}+\bar{x}}\right)}{p}\right) \\
& =\mathrm{e}\left(\frac{\bar{x}(s-r)}{p}\right) \sum_{\substack{j=1 \\
p \nmid \bar{j}+\bar{x}}}^{p-1} \mathrm{e}\left(\frac{r(\bar{j}+\bar{x})-s \bar{x}^{2} \overline{\bar{j}+\bar{x}}}{p}\right) \\
& =\mathrm{e}\left(\frac{\bar{x}(s-r)}{p}\right) \sum_{\substack{j=1 \\
j \neq \bar{x}}}^{p-1} \mathrm{e}\left(\frac{r j-s \bar{x}^{2} \bar{j}}{p}\right) \ll \sqrt{p} .
\end{aligned}
$$

Now we suppose $1 \leq u \leq p-1$. Then

$$
\Psi=\sum_{\substack{j=0 \\ \nmid \nmid a+j b \\ p \nmid a+j b+x}}^{p-1} \mathrm{e}\left(\frac{r(a+j b+x)+s(a+j b)+u j(a+j b)(a+j b+x)}{(a+j b)(a+j b+x) p}\right) .
$$


Let $g(j)=r(a+j b+x)+s(a+j b)+u j(a+j b)(a+j b+x)$, and $h(j)=$ $(a+j b)(a+j b+x)$. Since $1 \leq u, b \leq p-1$, we have $0<\operatorname{deg}(h)<\operatorname{deg}(g)$. That is to say, the rational function $g / h$ over $\mathbb{F}_{p}$ is not constant. Then Lemma 2.2 yields the assertion.

Lemma 2.4. For $1 \leq r_{1}, s_{1}, r_{2}, s_{2}, x \leq p-1,1 \leq d_{1}<d_{2} \leq p-1$ and $1 \leq u \leq p$, we have

$$
\begin{aligned}
\Omega= & \sum_{\substack{n=1 \\
p \nmid n+d_{1}, n+d_{1}+x \\
p \nmid n+d_{2}, n+d_{2}+x}}^{p} \mathrm{e}\left(\frac{r_{1} \overline{n+d_{1}}+s_{1} \overline{n+d_{1}+x}+r_{2} \overline{n+d_{2}}+s_{2} \overline{n+d_{2}+x}+u n}{p}\right) \\
& \ll \sqrt{p} .
\end{aligned}
$$

Proof. Let

$$
\begin{aligned}
g(n)= & r_{1}\left(n+d_{1}+x\right)\left(n+d_{2}\right)\left(n+d_{2}+x\right)+s_{1}\left(n+d_{1}\right)\left(n+d_{2}\right)\left(n+d_{2}+x\right) \\
& +r_{2}\left(n+d_{1}\right)\left(n+d_{1}+x\right)\left(n+d_{2}+x\right) \\
& +s_{2}\left(n+d_{1}\right)\left(n+d_{1}+x\right)\left(n+d_{2}\right) \\
& +u n\left(n+d_{1}\right)\left(n+d_{1}+x\right)\left(n+d_{2}\right)\left(n+d_{2}+x\right) \\
h(n)= & \left(n+d_{1}\right)\left(n+d_{1}+x\right)\left(n+d_{2}\right)\left(n+d_{2}+x\right) .
\end{aligned}
$$

We have

$$
\Omega=\sum_{\substack{n=1 \\ p \nmid n+d_{1}, n+d_{1}+x \\ p \nmid n+d_{2}, n+d_{2}+x}}^{p} \mathrm{e}\left(\frac{g(n)}{h(n) p}\right) .
$$

If $1 \leq u \leq p-1$, then $0<\operatorname{deg}(h)<\operatorname{deg}(g)$ and it remains to apply Lemma 2.2 .

If $u=p$, we need to prove $g(n) \not \equiv 0 \bmod p$. Suppose that $g(n) \equiv 0 \bmod p$. Then comparing the coefficients of $n^{3}, n^{2}, n$ and $n^{0}$ we have

$$
\left\{\begin{array}{l}
r_{1}+s_{1}+r_{2}+s_{2} \equiv 0 \bmod p \\
r_{1}\left(d_{1}+2 d_{2}+2 x\right)+s_{1}\left(d_{1}+2 d_{2}+x\right)+r_{2}\left(2 d_{1}+d_{2}+2 x\right) \\
\quad+s_{2}\left(2 d_{1}+d_{2}+x\right) \equiv 0 \bmod p \\
r_{1}\left(2 d_{1} d_{2}+d_{2}^{2}+d_{1} x+3 d_{2} x+x^{2}\right)+s_{1}\left(2 d_{1} d_{2}+d_{2}^{2}+d_{1} x+d_{2} x\right) \\
\quad+r_{2}\left(2 d_{1} d_{2}+d_{1}^{2}+3 d_{1} x+d_{2} x+x^{2}\right) \\
\quad+s_{2}\left(2 d_{1} d_{2}+d_{1}^{2}+d_{1} x+d_{2} x\right) \equiv 0 \bmod p, \\
r_{1}\left(d_{1} d_{2}^{2}+d_{1} d_{2} x+d_{2}^{2} x+d_{2} x^{2}\right)+s_{1}\left(d_{1} d_{2}^{2}+d_{1} d_{2} x\right) \\
\quad+r_{2}\left(d_{1}^{2} d_{2}+d_{1}^{2} x+d_{1} d_{2} x+d_{1} x^{2}\right)+s_{2}\left(d_{1}^{2} d_{2}+d_{1} d_{2} x\right) \equiv 0 \bmod p .
\end{array}\right.
$$


This gives

$$
\left\{\begin{array}{l}
r_{1}\left(d_{2}-d_{1}+x\right)+s_{1}\left(d_{2}-d_{1}\right)+r_{2} x \equiv 0 \bmod p \\
r_{1}\left(d_{2}^{2}-d_{1}^{2}+2 d_{2} x+x^{2}\right)+s_{1}\left(d_{2}^{2}-d_{1}^{2}\right)+r_{2} x\left(2 d_{1}+x\right) \equiv 0 \bmod p \\
r_{1}\left(d_{1} d_{2}^{2}-d_{1}^{2} d_{2}+d_{2}^{2} x+d_{2} x^{2}\right)+s_{1}\left(d_{1} d_{2}^{2}-d_{1}^{2} d_{2}\right)+r_{2} x\left(d_{1}^{2}+d_{1} x\right) \equiv 0 \bmod p
\end{array}\right.
$$

hence

$$
\left\{\begin{array}{l}
r_{1}\left(d_{1}^{2}+d_{2}^{2}-2 d_{1} d_{2}-d_{1} x+d_{2} x\right)+s_{1}\left(d_{1}^{2}+d_{2}^{2}-2 d_{1} d_{2}+d_{1} x-d_{2} x\right) \equiv 0 \bmod p \\
r_{1}\left(d_{1}^{3}-2 d_{1}^{2} d_{2}+d_{1} d_{2}^{2}-d_{1} d_{2} x+d_{2}^{2} x-d_{1} x^{2}+d_{2} x^{2}\right) \\
\quad+s_{1} d_{1}\left(d_{1}^{2}+d_{2}^{2}-2 d_{1} d_{2}+d_{1} x-d_{2} x\right) \equiv 0 \bmod p .
\end{array}\right.
$$

Therefore

$$
r_{1} x\left(d_{2}-d_{1}\right)\left(d_{2}-d_{1}+x\right) \equiv 0 \bmod p,
$$

and consequently $d_{2} \equiv d_{1}-x \bmod p$. Inserting this in (2.1), we have

$$
\left\{\begin{array}{l}
r_{1}+s_{1}+r_{2}+s_{2} \equiv 0 \bmod p \\
3 d_{1} r_{1}+\left(3 d_{1}-x\right) s_{1}+\left(3 d_{1}+x\right) r_{2}+3 d_{1} s_{2} \equiv 0 \bmod p \\
d_{1}^{2} r_{1}+\left(d_{1}-x\right)^{2} s_{1}+\left(d_{1}+x\right)^{2} r_{2}+d_{1}^{2} s_{2} \equiv 0 \bmod p \\
d_{1}^{2}\left(d_{1}-x\right) r_{1}+d_{1}\left(d_{1}-x\right)^{2} s_{1}+d_{1}\left(d_{1}^{2}+x^{2}\right) r_{2}+d_{1}^{2}\left(d_{1}-x\right) s_{2} \equiv 0 \bmod p
\end{array}\right.
$$

This implies $s_{1} \equiv r_{2} \equiv 0 \bmod p$, which is impossible. So $g(n) \not \equiv 0 \bmod p$, and an appeal to Lemma 2.2 completes the proof.

3. Proof of Theorem 1.1. For $a, b, t$ with $1 \leq a \leq a+(t-1) b \leq p-1$, by (1.1) we have

$$
\begin{aligned}
& \sum_{j=0}^{t-1} e_{a+j b}=\sum_{\substack{j=0 \\
p \nmid a+j b \\
p \nmid a+j b+x}}^{t-1}(-1)^{\overline{a+j b}+\overline{a+j b+x}}+O(1) \\
& =\frac{1}{p^{3}} \sum_{\substack{j=0 \\
p \nmid a+j b \\
p \nmid a+j b+x}}^{p-1} \sum_{l=0}^{t-1} \sum_{u=1}^{p} \mathrm{e}\left(\frac{u(j-l)}{p}\right) \sum_{c=1}^{p-1} \sum_{r=1}^{p} \mathrm{e}\left(\frac{r(\overline{a+j b}-c)}{p}\right) \\
& \times \sum_{d=1}^{p-1} \sum_{s=1}^{p} \mathrm{e}\left(\frac{s(\overline{a+j b+x}-d)}{p}\right)(-1)^{c+d}+O(1) \\
& =\frac{1}{p^{3}} \sum_{r=1}^{p-1} \sum_{s=1}^{p-1} \sum_{u=1}^{p} \sum_{l=0}^{t-1} \mathrm{e}\left(-\frac{u l}{p}\right) \sum_{c=1}^{p-1}(-1)^{c} \mathrm{e}\left(-\frac{r c}{p}\right) \sum_{d=1}^{p-1}(-1)^{d} \mathrm{e}\left(-\frac{s d}{p}\right) \\
& \times \sum_{\substack{j=0 \\
p \nmid a+j b \\
p \nmid a+j b+x}}^{p-1} \mathrm{e}\left(\frac{r \overline{a+j b}+s \overline{a+j b+x}+u j}{p}\right)+O(1) .
\end{aligned}
$$


Since

$$
\begin{aligned}
& \sum_{l=0}^{t-1} \mathrm{e}\left(-\frac{u l}{p}\right) \ll \frac{1}{|\sin (\pi u / p)|} \quad \text { for } p \nmid u, \\
& \sum_{c=1}^{p-1}(-1)^{c} \mathrm{e}\left(-\frac{r c}{p}\right) \ll \frac{1}{|\sin (\pi / 2-\pi r / p)|},
\end{aligned}
$$

from Lemma 2.3 we have

$$
\begin{aligned}
\sum_{j=0}^{t-1} e_{a+j b} & \ll \frac{t p^{1 / 2}}{p^{3}} \sum_{r=1}^{p-1} \frac{1}{|\sin (\pi / 2-\pi r / p)|} \sum_{s=1}^{p-1} \frac{1}{|\sin (\pi / 2-\pi s / p)|} \\
& +\frac{p^{1 / 2}}{p^{3}} \sum_{r=1}^{p-1} \frac{1}{|\sin (\pi / 2-\pi r / p)|} \sum_{s=1}^{p-1} \frac{1}{|\sin (\pi / 2-\pi s / p)|} \sum_{u=1}^{p-1} \frac{1}{|\sin (\pi u / p)|} \\
& \ll p^{1 / 2} \log ^{3} p .
\end{aligned}
$$

Therefore

$$
W\left(E_{p-1}\right)=\max _{a, b, t}\left|\sum_{j=0}^{t-1} e_{a+j b}\right| \ll p^{1 / 2} \log ^{3} p .
$$

For $0 \leq d_{1}<d_{2} \leq p-1-M$, from (1.1), (3.1) and Lemma 2.4 we have

$$
\sum_{n=1}^{M} e_{n+d_{1}} e_{n+d_{2}}=\sum_{\substack{n=1 \\ p \nmid n+d_{1}, n+d_{1}+x}}^{M}(-1)^{\overline{n+d_{1}}+\overline{n+d_{1}+x}+\overline{n+d_{2}}+\overline{n+d_{2}+x}}+O(1)
$$

$p \nmid n+d_{2}, n+d_{2}+x$

$$
=\frac{1}{p^{5}} \quad \sum_{n=1}^{p} \quad \sum_{l=1}^{M} \sum_{u=1}^{p} \mathrm{e}\left(\frac{u(n-l)}{p}\right) \sum_{c_{1}=1}^{p-1} \sum_{r_{1}=1}^{p} \mathrm{e}\left(\frac{r_{1}\left(\overline{n+d_{1}}-c_{1}\right)}{p}\right)
$$

$p \nmid n+d_{1}, n+d_{1}+x$

$p \nmid n+d_{2}, n+d_{2}+x$

$$
\begin{aligned}
& \times \sum_{d_{1}=1}^{p-1} \sum_{s_{1}=1}^{p} \mathrm{e}\left(\frac{s_{1}\left(\overline{n+d_{1}+x}-d_{1}\right)}{p}\right) \sum_{c_{2}=1}^{p-1} \sum_{r_{2}=1}^{p} \mathrm{e}\left(\frac{r_{2}\left(\overline{n+d_{2}}-c_{2}\right)}{p}\right) \\
& \times \sum_{d_{2}=1}^{p-1} \sum_{s_{2}=1}^{p} \mathrm{e}\left(\frac{s_{2}\left(\overline{n+d_{2}+x}-d_{2}\right)}{p}\right)(-1)^{c_{1}+d_{1}+c_{2}+d_{2}}+O(1) \\
& =\frac{1}{p^{5}} \sum_{r_{1}=1}^{p-1} \sum_{s_{1}=1}^{p-1} \sum_{r_{2}=1}^{p-1} \sum_{s_{2}=1}^{p-1} \sum_{u=1}^{p} \sum_{l=1}^{M} \mathrm{e}\left(-\frac{u l}{p}\right) \\
& \times \sum_{c_{1}=1}^{p-1}(-1)^{c_{1}} \mathrm{e}\left(-\frac{r_{1} c_{1}}{p}\right) \sum_{d_{1}=1}^{p-1}(-1)^{d_{1}} \mathrm{e}\left(-\frac{s_{1} d_{1}}{p}\right)
\end{aligned}
$$




$$
\begin{aligned}
& \times \sum_{c_{2}=1}^{p-1}(-1)^{c_{2}} \mathrm{e}\left(-\frac{r_{2} c_{2}}{p}\right) \sum_{d_{2}=1}^{p-1}(-1)^{d_{2}} \mathrm{e}\left(-\frac{s_{2} d_{2}}{p}\right) \\
& \times \quad \sum_{n=1}^{p} \mathrm{e}\left(\frac{r_{1} \overline{n+d_{1}}+s_{1} \overline{n+d_{1}+x}+r_{2} \overline{n+d_{2}}+s_{2} \overline{n+d_{2}+x}+u n}{p}\right) \\
& p \nmid n+d_{1}, n+d_{1}+x \\
& p \nmid n+d_{2}, n+d_{2}+x \\
& +O(1) \\
& \ll \frac{M p^{1 / 2}}{p^{5}} \sum_{r_{1}=1}^{p-1} \frac{1}{\left|\sin \left(\pi / 2-\pi r_{1} / p\right)\right|} \sum_{s_{1}=1}^{p-1} \frac{1}{\left|\sin \left(\pi / 2-\pi s_{1} / p\right)\right|} \\
& \times \sum_{r_{2}=1}^{p-1} \frac{1}{\left|\sin \left(\pi / 2-\pi r_{2} / p\right)\right|} \sum_{s_{2}=1}^{p-1} \frac{1}{\left|\sin \left(\pi / 2-\pi s_{2} / p\right)\right|} \\
& +\frac{p^{1 / 2}}{p^{5}} \sum_{r_{1}=1}^{p-1} \frac{1}{\left|\sin \left(\pi / 2-\pi r_{1} / p\right)\right|} \sum_{s_{1}=1}^{p-1} \frac{1}{\left|\sin \left(\pi / 2-\pi s_{1} / p\right)\right|} \\
& \times \sum_{r_{2}=1}^{p-1} \frac{1}{\left|\sin \left(\pi / 2-\pi r_{2} / p\right)\right|} \sum_{s_{2}=1}^{p-1} \frac{1}{\left|\sin \left(\pi / 2-\pi s_{2} / p\right)\right|} \sum_{u=1}^{p-1} \frac{1}{|\sin (\pi u / p)|} \\
& \ll p^{1 / 2} \log ^{5} p \text {. }
\end{aligned}
$$

Therefore

$$
C_{2}\left(E_{p-1}\right)=\max _{M, D}\left|\sum_{n=1}^{M} e_{n+d_{1}} e_{n+d_{2}}\right| \ll p^{1 / 2} \log ^{5} p
$$

For $1 \leq a+j b+d_{2} \leq p-1$ and $0 \leq d_{1}<d_{2}$, from (1.1) we have

$$
\begin{aligned}
& \sum_{j=0}^{t} e_{a+j b+d_{1}} e_{a+j b+d_{2}} \\
& =\sum_{\substack{j=0 \\
p \nmid a+j b+d_{1}, a+j b+d_{1}+x \\
p \nmid a+j b+d_{2}, a+j b+d_{2}+x}}^{t}(-1)^{\overline{a+j b+d_{1}}+\overline{a+j b+d_{1}+x}+\overline{a+j b+d_{2}}+\overline{a+j b+d_{2}+x}+O(1)} \\
& =\frac{1}{p^{5}} \sum_{\substack{p \nmid a+j b+d_{1}, a+j b+d_{1}+x \\
p \nmid a+j b+d_{2}, a+j b+d_{2}+x}}^{p} \sum_{l=0}^{t} \sum_{u=1}^{p} \mathrm{e}\left(\frac{u(j-l)}{p}\right) \sum_{c_{1}=1}^{p-1} \sum_{r_{1}=1}^{p} \mathrm{e}\left(\frac{r_{1}\left(\overline{a+j b+d_{1}}-c_{1}\right)}{p}\right) \\
& \quad \times \sum_{\substack{p-1 \\
d_{1}=1}}^{p} \mathrm{e}\left(\frac{s_{1}\left(\overline{a+j b+d_{1}=1}\right.}{p}\right) \sum_{c_{2}=1}^{p-1} \sum_{r_{2}=1}^{p} \mathrm{e}\left(\frac{r_{2}\left(\overline{a+j b+d_{2}}-c_{2}\right)}{p}\right)
\end{aligned}
$$




$$
\begin{aligned}
& \times \sum_{d_{2}=1}^{p-1} \sum_{s_{2}=1}^{p} \mathrm{e}\left(\frac{s_{2}\left(\overline{a+j b+d_{2}+x}-d_{2}\right)}{p}\right)(-1)^{c_{1}+d_{1}+c_{2}+d_{2}}+O(1) \\
= & \frac{1}{p^{5}} \sum_{r_{1}=1}^{p-1} \sum_{s_{1}=1}^{p-1} \sum_{r_{2}=1}^{p-1} \sum_{s_{2}=1}^{p-1} \sum_{u=1}^{p} \sum_{l=0}^{t} \mathrm{e}\left(-\frac{u l}{p}\right) \sum_{c_{1}=1}^{p-1}(-1)^{c_{1}} \mathrm{e}\left(-\frac{r_{1} c_{1}}{p}\right) \\
& \times \sum_{d_{1}=1}^{p-1}(-1)^{d_{1}} \mathrm{e}\left(-\frac{s_{1} d_{1}}{p}\right) \sum_{c_{2}=1}^{p-1}(-1)^{c_{2}} \mathrm{e}\left(-\frac{r_{2} c_{2}}{p}\right) \sum_{d_{2}=1}^{p-1}(-1)^{d_{2}} \mathrm{e}\left(-\frac{s_{2} d_{2}}{p}\right) \\
& \left.\times \sum_{j=0}^{p} \bar{p} \frac{r_{1} \overline{a+j b+d_{1}}+s_{1} \overline{a+j b+d_{1}+x}}{p}\right) \\
& \quad \times \mathrm{e}\left(\frac{r_{2} \overline{a+j b+d_{2}}+s_{2} \overline{a+j b+d_{2}+x}+u j}{p}\right)+O(1) .
\end{aligned}
$$

By Lemma 2.4 we get

$$
\sum_{j=0}^{p} \mathrm{e}\left(\frac{r_{1} \overline{a+j b+d_{1}}+s_{1} \overline{a+j b+d_{1}+x}}{p}\right)
$$

$p \nmid a+j b+d_{1}$

$p \nmid a+j b+d_{1}+x$

$p \nmid a+j b+d_{2}$

$p \nmid a+j b+d_{2}+x$

$$
\begin{aligned}
& \times \mathrm{e}\left(\frac{r_{2} \overline{a+j b+d_{2}}+s_{2} \overline{a+j b+d_{2}+x}+u j}{p}\right) \\
= & \mathrm{e}\left(-\frac{u a \bar{b}}{p}\right) \\
& \times \sum_{\substack{j=0 \\
p \nmid j+d_{1}, j+d_{1}+x \\
p \nmid j+d_{2}, j+d_{2}+x}} \mathrm{e}\left(\frac{r_{1} \overline{j+d_{1}}+s_{1} \overline{j+d_{1}+x}+r_{2} \overline{j+d_{2}}+s_{2} \overline{j+d_{2}+x}+u \bar{b} j}{p}\right)
\end{aligned}
$$

$\ll p^{1 / 2}$.

Then from (3.1) we have

$$
\begin{aligned}
\sum_{j=0}^{t} e_{a+j b+d_{1}} e_{a+j b+} & d_{2} \\
\ll & \frac{t p^{1 / 2}}{p^{5}} \sum_{r_{1}=1}^{p-1} \frac{1}{\left|\sin \left(\pi / 2-\pi r_{1} / p\right)\right|} \sum_{s_{1}=1}^{p-1} \frac{1}{\left|\sin \left(\pi / 2-\pi s_{1} / p\right)\right|} \\
& \times \sum_{r_{2}=1}^{p-1} \frac{1}{\left|\sin \left(\pi / 2-\pi r_{2} / p\right)\right|} \sum_{s_{2}=1}^{p-1} \frac{1}{\left|\sin \left(\pi / 2-\pi s_{2} / p\right)\right|}
\end{aligned}
$$




$$
\begin{aligned}
& +\frac{p^{1 / 2}}{p^{5}} \sum_{r_{1}=1}^{p-1} \frac{1}{\left|\sin \left(\pi / 2-\pi r_{1} / p\right)\right|} \sum_{s_{1}=1}^{p-1} \frac{1}{\left|\sin \left(\pi / 2-\pi s_{1} / p\right)\right|} \\
& \times \sum_{r_{2}=1}^{p-1} \frac{1}{\left|\sin \left(\pi / 2-\pi r_{2} / p\right)\right|} \sum_{s_{2}=1}^{p-1} \frac{1}{\left|\sin \left(\pi / 2-\pi s_{2} / p\right)\right|} \sum_{u=1}^{p-1} \frac{1}{|\sin (\pi u / p)|} \\
& \ll p^{1 / 2} \log ^{5} p .
\end{aligned}
$$

\section{Therefore}

$$
Q_{2}\left(E_{p-1}\right)=\max _{a, b, t, D}\left|\sum_{j=0}^{t} e_{a+j b+d_{1}} e_{a+j b+d_{2}}\right| \ll p^{1 / 2} \log ^{5} p .
$$

This completes the proof of Theorem 1.1.

\section{References}

[1] J. Cassaigne, S. Ferenczi, C. Mauduit, J. Rivat and A. Sárközy, On finite pseudorandom binary sequences III: The Liouville function, I, Acta Arith. 87 (1999), 367-390.

[2] - , - - - - - On finite pseudorandom binary sequences IV: The Liouville function, II, ibid. 95 (2000), pp. 343-359.

[3] J. Cassaigne, C. Mauduit and A. Sárközy, On finite pseudorandom binary sequences VII: The measures of pseudorandomness, ibid. 103 (2002), 97-118.

[4] E. Fouvry, P. Michel, J. Rivat and A. Sárközy, On the pseudorandomness of the signs of Kloosterman sums, J. Austral. Math. Soc. 77 (2004), 425-436.

[5] C. Mauduit and A. Sárközy, On finite pseudorandom binary sequences I: Measure of pseudorandomness, the Legendre symbol, Acta Arith. 82 (1997), 365-377.

[6] - - - On the measures of pseudorandomness of binary sequences, Discrete Math. 271 (2003), 195-207.

[7] - - - Construction of pseudorandom binary sequences by using the multiplicative inverse, Acta Math. Hungar. 108 (2005), 239-252.

[8] C. J. Moreno and O. Moreno, Exponential sums and Goppa codes: I, Proc. Amer. Math. Soc. 111 (1991), 523-531.

[9] A. Weil, Sur les courbes algébriques et les variétés qui s'en déduisent, Act. Sci. Ind. 1041, Hermann, Paris, 1948.

Department of Mathematics

Northwest University

Xi'an, Shaanxi, P.R. China

E-mail: hnliu@nwu.edu.cn

Received on 9.11.2005

and in revised form on 1.8.2006 